\title{
A Rare Adverse Effect of Anti-Tumor Necrosis Factor Alpha Therapy: Sarcoidosis
}

\author{
Gönen MENGİ, ${ }^{1}$ Feride GÖĞÜŞ² \\ ${ }^{1}$ Department of Rheumatology, Muğla Sitkı Koçman University, Education and Research Hospital, Muğla, Turkey \\ ${ }^{2}$ Department of Physical Medicine and Rehabilitation, Medical Faculty of Gazi University, Ankara, Turkey
}

\begin{abstract}
Anti-tumor necrosis factor alpha (anti-TNF- $\alpha$ ) therapy has been widely used for the management of rheumatologic diseases. The most frequent adverse effects of anti-TNF- $\alpha$ therapy are infections and malignancies while sarcoidosis is a rare condition. On the other hand, anti-TNF- $\alpha$ therapy has been used in the treatment of sarcoidosis. Elucidation of this paradoxical issue is unclear. In this article, we report an ankylosing spondylitis patient who was diagnosed as sarcoidosis during the period of etanercept usage. Sarcoidosis as a possible adverse effect should be kept in mind during anti-TNF- $\alpha$ therapy.

Keywords: Adverse effect, anti-tumor necrosis factor alpha therapy, sarcoidosis.
\end{abstract}

Anti-tumor necrosis factor alpha (anti-TNF- $\alpha$ ) therapy has been widely used around the world for the management of ankylosing spondylitis (AS) and rheumatoid arthritis for many years. The most avoided adverse effects of anti-TNF- $\alpha$ therapy include infections like tuberculosis and malignancies. ${ }^{1,2}$ However, granulomatous conditions such as sarcoidosis have scarcely been reported in the literature. ${ }^{3}$ The frequency of this adverse effect has been reported as $0.04 \%$ $(1 / 2800) .{ }^{4}$ Etanercept is the most frequently mentioned agent followed by infliximab and adalimumab. ${ }^{5}$ The pathogenesis of this issue is not clear yet, ${ }^{3}$ but an imbalance of cytokines towards interferons and chemokines and probably interleukin-17 has been implicated. ${ }^{6}$

Tumor necrosis factor-alpha plays a role in the development of granulomatous diseases such as sarcoidosis. ${ }^{7}$ On the other hand, infliximab, an anti-TNF- $\alpha$ monoclonal antibody, has been shown to be effective in the treatment of pulmonary sarcoidosis while etanercept, a soluble TNF- $\alpha$ receptor fusion protein, has not. ${ }^{3}$ Moreover, in a study including 17 patients with pulmonary sarcoidosis, etanercept treatment was found to be associated with aggravation of the disease. ${ }^{8}$ In this article, we report an AS patient who was diagnosed as sarcoidosis during the period of etanercept usage.

\section{CASE REPORT}

A 29-year-old male patient who had AS for 13 years applied to our rheumatology outpatient clinic for routine yearly control. His AS had been under control with etanercept for 43 months. After the routine follow-up evaluation, a thorax computed tomography scan was performed because of hilar enlargement in the chest X-ray. 
Multiple lymphadenopathies, the largest one with $2 \mathrm{~cm}$ in diameter, were detected in computed tomography scan (Figure 1).

Lymph node biopsy was performed by broncoscopy and endobronchial ultrasonography. Biopsy result was compatible with granulomatous disease. All the infectious studies performed on pulmonary lymph node tissues were negative. Angiotensin converting enzyme level was at the upper limit with a value of $47.6 \mathrm{U} / \mathrm{L}$ (normal: 8-52 U/L). Sarcoidosis was found to be compatible with the condition after excluding tuberculosis. Etanercept was ceased following the diagnosis of sarcoidosis. No additional medical treatment was suggested for sarcoidosis. Nonsteroid anti-inflammatory drugs were prescribed for the control of AS. Five months later, followup thorax computed tomography scan showed resolution of the mediastinal lymphadenopathies (Figure 2).

However, pain, dysfunction in daily living activities related with AS, high disease activity markers (erythrocyte sedimentation rate: $53 \mathrm{~mm}$ /hour, C-reactive protein: $40 \mathrm{mg} / \mathrm{L}$ [normal: 0-6 mg/L], and Bath Ankylosing Spondylitis Disease Activity Index of 6.3 [normal: 0-4]) during the period without etanercept prompted us to consider another anti-TNF- $\alpha$ agent. At the seventh month of etanercept cessation, golimumab with the dosage of $50 \mathrm{mg} /$ month was commenced to compete with the symptoms and findings associated with AS. At the ninth month of

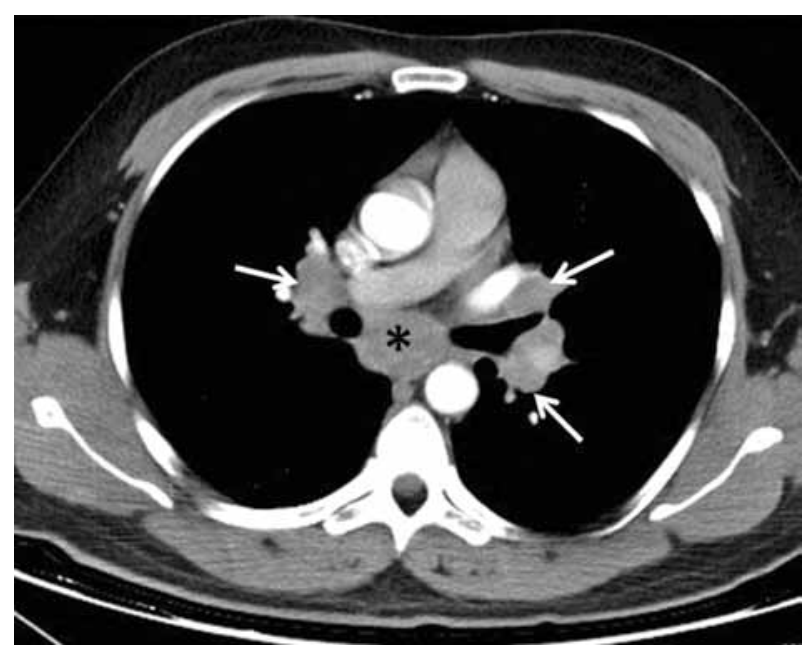

Figure 1. Lymphadenopathies at both hiluses (arrows) and subcarinal area (asterisk). follow-up period after inception of golimumab, the patient had no pain with normal disease activity markers. There were no pathological findings regarding sarcoidosis in the examination of the pulmonary system (Figure 3). A written informed consent was obtained from the patient.

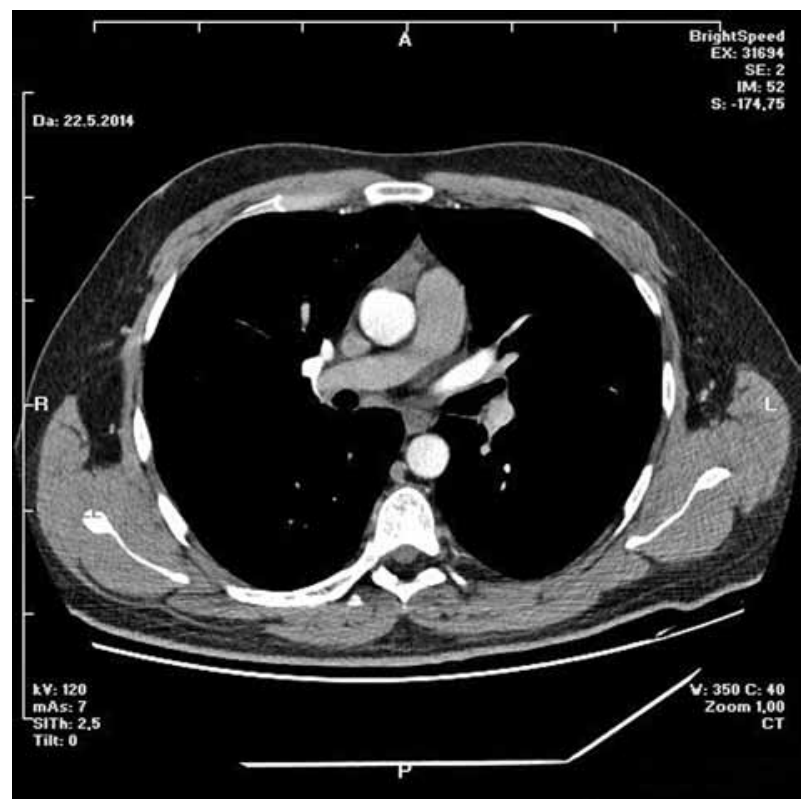

Figure 2. Normal thorax computed tomography scan five months after discontinuation of etanercept.

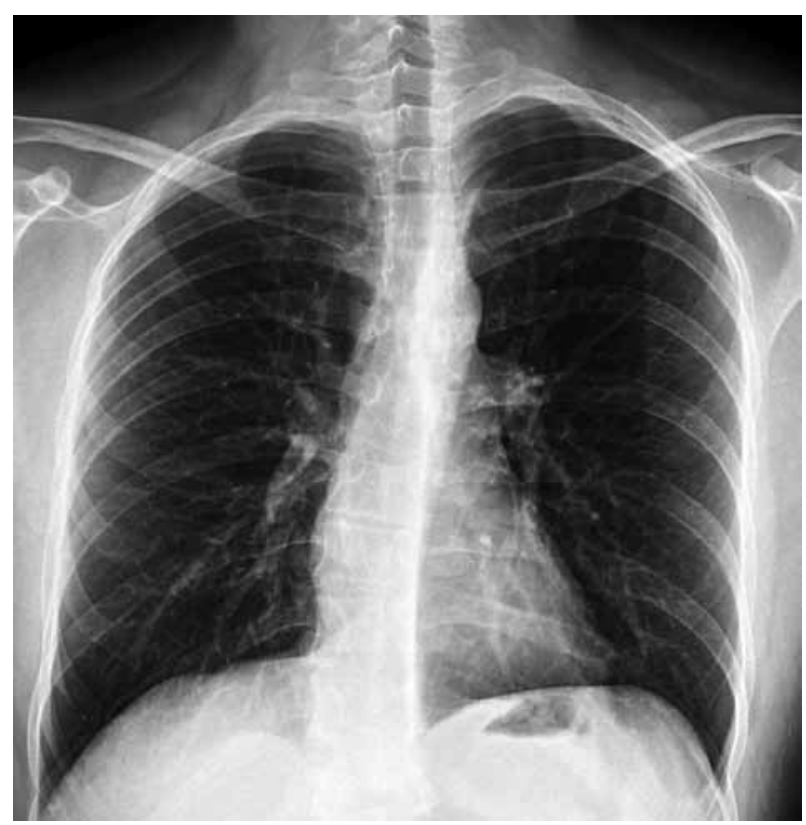

Figure 3. Normal chest X-ray at ninth month of initiation of golimumab. 


\section{DISCUSSION}

Sarcoidosis is a multisystem disease characterized by noncaseating granulomatous infiltration primarily of the lungs and the lymphatic system. ${ }^{9}$ The etiology of sarcoidosis remains unclear. Exacerbated immune response due to antigenic stimuli of infections, environmental factors or autoantigens have been accused. ${ }^{10}$

Tumor necrosis factor-alpha plays a role in forming the inflammatory granuloma. Anti-TNF- $\alpha$ therapy is considered to treat sarcoidosis by blocking TNF- $\alpha$ action. ${ }^{10}$ Paradoxically, sarcoidosis cases developing during anti-TNF- $\alpha$ treatment have been reported. ${ }^{10,11}$ This conflicting issue has not been clarified yet. Etanercept, infliximab, and adalimumab were found to be the most common agents, respectively, to be responsible for this condition. ${ }^{10}$ In most of the cases in which sarcoidosis developed, etanercept emerged as the responsible anti-TNF- $\alpha$ agent. ${ }^{11}$ However, although scarce, there are some case reports related with infliximab and adalimumab. ${ }^{11}$ The reason of this issue may be the different molecular structure of etanercept. ${ }^{10-12}$ Etanercept is a soluble receptor fusion protein. ${ }^{13}$ TNF- $\alpha$ inhibition by etanercept is limited, ${ }^{10-12}$ since it binds only to soluble TNF- $\alpha$. Limited blocking of TNF- $\alpha$ by etanercept can lead to redistribution of TNF- $\alpha$ in low-concentrated regions like lungs. ${ }^{10}$ Nonetheless, since sarcoidlike reactions have also been reported during the treatment with monoclonal anti-TNF- $\alpha$ agents like infliximab and adalimumab, other mechanisms should also be considered. Infliximab is a chimeric monoclonal antibody directed against TNF- $\alpha$. Adalimumab is a recombinant fully human monoclonal antibody against TNF- $\alpha$. Golimumab is a human immunoglobulin G1 kappa monoclonal antibody specific for TNF- $\alpha .{ }^{13}$ They bind to both soluble and membrane bounded TNF- $\alpha$ molecule. However, etanercept targets soluble TNF- $\alpha$ distinctly. Anti-TNF- $\alpha$ agents can change the cytokine environment. Etanercept can reinforce $\mathrm{T}$-cell production of interferon gamma; furthermore, monoclonal anti-TNF- $\alpha$ antibodies can increase $\mathrm{T}$ helper 1 : $\mathrm{T}$ helper 2 ratio which enhances interferon gamma production. Activated interferon gamma production, an important cytokine in granuloma formation, may contribute to the tendency to develop sarcoid-like reactions. Besides, these granulomatous reactions mainly occur in the lungs which was the case in our patient, and/or the skin, which are both exposed to exogenous antigens. In patients under anti-TNF- $\alpha$ treatment, some infections such as Mycobacterium tuberculosis and Propionibacterium acnes can trigger granuloma formation. ${ }^{4}$

Usually, clinical or radiologic symptoms resolve after cessation of anti-TNF- $\alpha$ agent as was observed in our case. ${ }^{12}$ Occasionally, in serious cases, steroid treatment may be necessary for resolving the clinical condition. ${ }^{4,11}$ Sometimes a new anti-TNF- $\alpha$ agent may be necessary to overcome the burden of the rheumatologic condition. Sarcoidosis related with anti-TNF- $\alpha$ treatment may not emerge with this new agent; however, sarcoid tissue might reoccur in some cases. ${ }^{4}$ In these conditions, cautious monitoring is essential regarding the development of recurrent sarcoid reaction. Thus, granulomatous diseases such as sarcoidosis as an adverse effect should be kept in mind during anti-TNF- $\alpha$ therapy.

\section{Declaration of conflicting interests}

The authors declared no conflicts of interest with respect to the authorship and/or publication of this article.

\section{Funding}

The authors received no financial support for the research and/or authorship of this article.

\section{REFERENCES}

1. Papamichael K, Mantzaris GJ, Peyrin-Biroulet L. A safety assessment of anti-tumor necrosis factor alpha therapy for treatment of Crohn's disease. Expert Opin Drug Saf 2016;15:493-501.

2. Sampaio-Barros PD, van der Horst-Bruinsma IE2. Adverse effects of TNF inhibitors in SpA: are they different from RA? Best Pract Res Clin Rheumatol 2014;28:747-63.

3. Thongpooswan S, Abrudescu A. Lung sarcoidosis in etanercept treated rheumatoid arthritis patient: a case report and review of the literature. Case Rep Rheumatol 2014;2014:358567.

4. Daïen CI, Monnier A, Claudepierre P, Constantin A, Eschard JP, Houvenagel E, et al. Sarcoid-like granulomatosis in patients treated with tumor necrosis factor blockers: 10 cases. Rheumatology (Oxford) 2009;48:883-6.

5. Gifre L, Ruiz-Esquide V, Xaubet A, Gómez-Puerta JA, Hernández MV, Sanmartí R. Lung sarcoidosis 
induced by TNF antagonists in rheumatoid arthritis: a case presentation and a literature review. Arch Bronconeumol 2011;47:208-12.

6. Wendling D, Prati C. Paradoxical effects of anti-TNF- $\alpha$ agents in inflammatory diseases. Expert Rev Clin Immunol 2014;10:159-69.

7. Antoniu SA. Targeting the TNF-alpha pathway in sarcoidosis. Expert Opin Ther Targets 2010;14:219.

8. Utz JP, Limper AH, Kalra S, Specks U, Scott JP, VukPavlovic Z, et al. Etanercept for the treatment of stage II and III progressive pulmonary sarcoidosis. Chest 2003;124:177-85.

9. González-López MA, Blanco R, González-Vela MC, Fernández-Llaca H, Rodríguez-Valverde V. Development of sarcoidosis during etanercept therapy. Arthritis Rheum 2006;55:817-20.
10. Unterstell N, Bressan AL, Serpa LA, Fonseca e Castro PP, Gripp AC. Systemic sarcoidosis induced by etanercept: first Brazilian case report. An Bras Dermatol 2013;88:197-9.

11. Akiyama M, Kaneko Y, Hanaoka H, Kuwana M, Takeuchi T. Acute kidney injury due to renal sarcoidosis during etanercept therapy: a case report and literature review. Intern Med 2015;54:1131-4.

12. Ishiguro T, Takayanagi N, Kurashima K, Matsushita A, Harasawa K, Yoneda K, et al. Development of sarcoidosis during etanercept therapy. Intern Med 2008;47:1021-5.

13. Available from: https://www.uptodate.com/contents/ overview-of-biologic-agents-in-the-rheumatic-disease s?source $=$ machineLearning \&search=etanercept \&sel ectedTitle $=6 \% 7 \mathrm{E} 150$ \& sectionRank $=1 \&$ anchor $=\mathrm{H} 13$ \#H13, [Access: June 28, 2016]. 